\title{
Directional Random Scattering MIMO Channels: Entropy Analysis and Capacity Optimization
}

\author{
Parastoo Sadeghi, Thushara D. Abhayapala, and Rodney A. Kennedy ${ }^{\dagger}$ \\ Research School of Information Sciences and Engineering (RSISE) \\ The Australian National University \\ Canberra ACT 0200 Australia \\ Emails: $\{$ parastoo.sadeghi $\} ;\{$ thushara.abhayapala $\} ;\{$ rodney.kennedy $\} @$ anu.edu.au
}

\begin{abstract}
In this paper, we study the effect of directional random scattering on the capacity of multiple-input multiple-output (MIMO) systems. First, we use the spatial decomposition of the MIMO channel matrix to analyze the randomness (entropy) of directional scattering. The analysis shows that directional scatterers (with at least a null in the angular power spectrum) will no longer be random when the receiver observation radius is sufficiently large. Therefore, directional scattering limits the expected linear increase of MIMO capacity with increasing the number of antennas. Second, we consider the effect of receiver antenna arrangement (positions) on the capacity of MIMO systems. For any random scatterer with a given angular power spectrum, we show that it is possible to choose the receiver antenna arrangement with the optimum whitening of the MIMO channel matrix that, in turn, maximizes MIMO channel capacity.
\end{abstract}

\section{INTRODUCTION}

\section{A. Motivation and Background}

The pioneering work of Telatar [1], and Foschini and Gans [2] predicted linear capacity enhancement in multiple-input multiple-output (MIMO) systems by increasing the number of transmitter/receiver antenna elements. However, this analysis assumes independent fading paths between the transmitter and receiver antenna elements. In practical systems, the assumption of independent fading may be violated due to insufficient antenna spacing and restricted/directional angular spread of scatterers [2]. The effect of fading correlation on MIMO capacity has been studied in the literature, where channel correlation is often decomposed into the transmitter/receiver correlation matrices [3]-[7]. A limitation of this approach is that the effects of random scattering environment and deterministic antenna configurations are intertwined in the correlation matrices [8]. This often hinders obtaining a generic insight into the effect of directional scattering on the capacity of MIMO systems and the possibility of MIMO capacity optimization by using the optimum antenna configuration.

Accurate physical modeling of the MIMO channel matrix requires that the scattering characteristics of the wireless spatial channel be taken into account. In [9], Muller proposed the decomposition of the MIMO channel based on the random propagation from transmitter antennas to the scattering objects

\footnotetext{
†Thushara Abhayapala and Rodney Kennedy are also associated with the National ICT Australia (NICTA). This work was supported by the ARC
} Discovery Project under the grant number DP0343804. and from the scattering objects to receiver antennas. The generality of this model and other models was further studied in [10] based on the maximum entropy principle. A spatial model for MIMO channels was introduced by Abhayapala et al. [11] by decomposing the MIMO channel matrix into the product of three distinct factors: 1) a deterministic receiver configuration matrix $\mathbf{J}_{R}, 2$ ) a random scattering matrix $\mathbf{H}_{s}$, and 3) a deterministic transmitter configuration matrix $\mathbf{J}_{T}$. This model is different from those surveyed in [10], in the sense that it is directly derived from the solution to the farfield wave propagation equation. This, in turn, enables a general and novel analysis of the effect of random scattering fields, without direct involvement of the geometry of scatterers. Moreover, it enables optimizing the transmitter/receiver antenna configurations to maximize MIMO channel capacity.

\section{B. Problem Statement and Contributions}

As a possible extension of the previous work in [8], [11], the following question arises: "For a given random scattering environment, how does MIMO receiver antenna configuration (including radius, number, and arrangement) affect the MIMO channel capacity?"

In this paper, we aim to answer the above question by providing the following contributions:

1) In Section II, we show that optimizing MIMO capacity is directly related to maximizing the randomness (entropy) of scatterers, as observed by the receiver sensors. This is equivalent to (ideally) whitening of MIMO channel matrix. While establishing this link is not new by itself, application of this concept together with the spatial decomposition of MIMO channel matrix [11] is novel.

2) In Section III, we study the inherent randomness (entropy) of the scattering field, as observed in a scattererfree region around the receiver antenna elements. The highlight of this analysis is that a directional scattering field (with at least a single null in its angular power spectrum) will no longer be random when the receiver observation radius is sufficiently large. Therefore, directional scattering limits the expected linear increase of MIMO capacity with increasing the number of antennas.

3) In Section IV, we show that it is possible to optimize MIMO channel capacity by proper receiver antenna 
arrangement. For a given random scatterer with a known angular power spectrum, the optimum antenna arrangement transforms the random scatterer to minimize the correlation in the MIMO channel matrix. This proposition is validated in Sections IV and V by numerical analysis.

\section{MIMO CHANNEL CAPACITY}

We start with the classical MIMO system model with $N_{R}$ receiver and $N_{T}$ transmitter antenna elements given as [1]

$$
\mathbf{r}=\mathbf{H} \mathbf{x}+\mathbf{n},
$$

where $\mathbf{r}$ is the $N_{R} \times 1$ received signal vector, $\mathbf{H}$ is the $N_{R} \times N_{T}$ random channel gain matrix and the element $h_{r, t}$ denotes channel gain from the $t^{t h}$ transmitter to the $r^{t h}$ receiver antenna, $\mathbf{x}$ is the $N_{T} \times 1$ transmitted signal vector with total power $E\left\{\mathbf{x x}^{H}\right\}=P$, where $E\{\cdot\}$ and superscript $H$ denote expectation and Hermitian transpose, respectively. $\mathbf{n}$ in (1) is the $N_{R} \times 1$ noise vector that is assumed to be additive white Gaussian noise (AWGN) with variance per dimension $\sigma^{2}$ and independent among the receiver antenna elements. It is noted that usually in the literature, the elements of channel matrix $\mathbf{H}$ are assumed to be complex Gaussian [1].

When the transmitted signal vector $\mathbf{x}$ contains Gaussiandistributed, statistically independent, and equal power components, the (constrained) ergodic MIMO channel capacity is given by [1]

$$
C=E_{\mathbf{H}}\left\{\log \left|\mathbf{I}_{N_{R}}+\frac{\eta}{N_{T}} \mathbf{H} \mathbf{H}^{H}\right|\right\},
$$

where $|\cdot|$ denotes the determinant of a matrix, $\eta \triangleq P / 2 \sigma^{2}$ is proportional to the signal to noise ratio ( $\mathrm{SNR}$ ), and the expectation is over all possible realizations of the random channel matrix $\mathbf{H}$. Also, $\mathbf{I}_{N_{R}}$ is the $N_{R} \times N_{R}$ square identity matrix.

The MIMO capacity in (2) can be upper bounded as

$$
C \leqslant \log \left|\mathbf{I}_{N_{R}}+\frac{\eta}{N_{T}} E_{\mathbf{H}}\left\{\mathbf{H H}^{H}\right\}\right|,
$$

where (3) follows from the concavity of $\log |\cdot|$ function [12, pp. 24-25]. Since the channel covariance matrix $\mathbf{W} \triangleq$ $\frac{\eta}{N_{T}} E_{\mathbf{H}}\left\{\mathbf{H H}^{H}\right\}$ is a non-negative definite and Hermitian symmetric matrix, we can use Hadamard's inequality [12, p. 233] in (3) as follows

$$
\left|\mathbf{I}_{N_{R}}+\mathbf{W}\right| \leqslant \prod_{i=1}^{N_{R}}\left(1+W_{i i}\right),
$$

where the equality holds iff the covariance matrix $\mathbf{W}$ is diagonal, i.e, where the random variables (r.v) in the matrix $\left(\mathbf{H H}^{H}\right)$ are uncorrelated from each other. In other words, the covariance matrix $\mathbf{W}$ has a key role in the MIMO capacity. Therefore, we provide the following proposition:

Proposition 1: The MIMO channel covariance matrix $\mathbf{W} \triangleq$ $\frac{\eta}{N_{T}} E_{\mathbf{H}}\left\{\mathbf{H H}^{H}\right\}$ is controllable by means of proper antenna configuration. Maximizing $|\mathbf{W}|$ or decorrelating $\left(\mathbf{H H}^{H}\right)$ maximizes the MIMO capacity upper bound in (3) and hence, can be targeted to optimize MIMO capacity.
Remark: It was shown in [8] that the upper bound in (3) is an accurate estimate of the MIMO capacity when $N_{T}>>N_{R}$ and the transmitted branches are uncorrelated. This condition is satisfied in many practical wireless scenarios and the Proposition 1 is accurate. In other cases, decorrelating $\left(\mathbf{H H}^{H}\right)$ may not be equivalent to capacity optimization ${ }^{1}$ and the optimum antenna configuration should be chosen to directly maximize the MIMO capacity expression in (2). Nevertheless, it is instructive to examine the properties of $|\mathbf{W}|$. Since the elements in the channel matrix $\mathbf{H}$ are Gaussian, $|\mathbf{W}|$ is closely related to the entropy (randomness) of $\mathbf{H}[12$, pp. 230-231]. Therefore, in Section III, we first analyze the entropy of the MIMO channel matrix by using its spatial decomposition [11].

\section{RANDOM SCATTERER ENTROPY}

\section{A. Preliminaries}

We start this section by borrowing the main results in [11], which are essential for our analysis. Consider a scattering model, which consists of two scatterer-free spheres around the transmitter and receiver antenna arrays with radii $r_{T}$ and $r_{R}$, respectively and a scattering field in between. We refer to $r_{R}$ and $r_{T}$ as receiver radius (size) and transmitter radius (size), respectively. It was shown in [11] that the MIMO channel matrix $\mathbf{H}$ in (1) may be decomposed into the following product

$$
\mathbf{H}=\mathbf{J}_{R} \mathbf{H}_{s} \mathbf{J}_{T}^{H},
$$

where $\mathbf{J}_{T}$ and $\mathbf{J}_{R}$ denote deterministic transmitter and receiver antenna configuration matrices, respectively and $\mathbf{H}_{s}$ is the random scattering matrix. The decomposition of $\mathbf{H}$ in (5) bears a different physical meaning from those proposed in [3]-[7] and surveyed in [10]. For example, $\mathbf{J}_{T}$ and $\mathbf{J}_{R}$ do not represent any transmitter or receiver fading correlations and $\mathbf{H}_{s}$ does not necessarily consist of i.i.d random variables. The elements of matrices $\mathbf{J}_{T}$ and $\mathbf{J}_{R}$ in (5) only depend on the physical antenna positions and not on the relative distance between scatterers and antennas as in [9].

For simpler, yet still insightful analysis, we assume that the multipath propagation is restricted to the horizontal plane. However, our approach may be generalized to cover 3-D scattering. Moreover, we assume that $N_{T}>>N_{R}$ and the transmitted branches are uncorrelated. In this case, it suffices to consider the vector of channel gains from a single and representative transmitter antenna and its associated covariance matrix at the receiver side [8]. With this assumption, $\mathbf{J}_{T}=1$ is a unity scaler, $\mathbf{H}_{s}$ reduces to a vector, and the channel matrix $\mathbf{H}$ in (5) is rewritten as

$$
\mathbf{H}=\mathbf{J}_{R} \mathbf{H}_{s} .
$$

In the decomposition of channel matrix in (6), the only random component is the scattering vector $\mathbf{H}_{s}\left(\mathbf{J}_{R}\right.$ is fixed for a given receiver antenna configuration). Therefore, we examine the randomness of $\mathbf{H}_{s}$ in more detail. Assume that the signal that enters the receiver scatterer-free region at an angle $\phi$

\footnotetext{
${ }^{1}$ It was shown in [13] that for $N_{T}=N_{R}=2$, while transmitter and receiver correlations always decrease the ergodic capacity, possible presence of anti-diagonal correlations have a positive impact on the capacity.
} 
experiences a complex random scattering gain $A(\phi)$. The elements of $\mathbf{H}_{s}$ are the Fourier series coefficients of $A(\phi)$ [11]. That is,

$$
\mathbf{H}_{s}=\left[\begin{array}{lll}
\beta_{-M_{R}} & \cdots & \beta_{M_{R}}
\end{array}\right]^{T},
$$

where

$$
\beta_{m}=\frac{1}{2 \pi} \int_{0}^{2 \pi} A(\phi) e^{-i m \phi} d \phi .
$$

$\mathbf{H}_{s}$ in (7) is a $\left(2 M_{R}+1\right) \times 1$ vector, where $M_{R}$ is directly proportional to the receiver radius encompassing receiver antenna elements $r_{R}$ [11], which is given as

$$
M_{R}=\left\lceil\frac{k e r_{R}}{2}\right\rceil,
$$

where $\lceil$.$\rceil denotes the ceiling operator, k=2 \pi / \lambda$, and $\lambda$ is the wavelength.

Furthermore, we assume uncorrelated scattering, which means that the complex random gains $A(\phi)$ and $A\left(\phi^{\prime}\right)$ at two distinct incident angles are uncorrelated from each other and the angular power spectrum $P(\phi)$ is defined as

$$
E\left\{A(\phi) A^{*}\left(\phi^{\prime}\right)\right\} \triangleq\left\{\begin{array}{cc}
P(\phi) & \phi=\phi^{\prime} \\
0 & \text { otherwise }
\end{array} .\right.
$$

For our analysis, we do not need to assume a specific scattering geometry. The combined effect of scattering objects between the transmitter and the receiver is manifested through the random scattering gain $A(\phi)$ and its angular power spectrum $P(\phi)$ as a function of observation azimuth angle $\phi$ at the receiver. Directional scattering refers to the case where $P(\phi)$ is non-zero for a limited angular range between $[0,2 \pi)$.

\section{B. Entropy of $H_{s}$}

Using (8), (10), and following a few intermediate steps, the cross-correlation of $\beta_{m}$ and $\beta_{n}$ in (7) is derived as

$$
E\left\{\beta_{m} \beta_{n}^{*}\right\}=\gamma_{m-n},
$$

where $\gamma_{m-n}$ is the $(m-n)^{t h}$ Fourier series coefficient of the angular power spectrum

$$
\gamma_{m-n}=\frac{1}{2 \pi} \int_{0}^{2 \pi} P(\phi) e^{-i(m-n) \phi} d \phi .
$$

We denote the covariance matrix of $\mathbf{H}_{s}$ as $\boldsymbol{\Gamma}=E\left\{\mathbf{H}_{s} \mathbf{H}_{s}^{H}\right\}$, with elements given by (12). Assuming that the real and imaginary parts of $A(\phi)$ are uncorrelated, the entropy of the $\left(2 M_{R}+1\right) \times 1$ complex-valued and multivariate Gaussian scattering vector $\mathbf{H}_{s}$ is given as [12, p. 230]

$$
h\left(\mathbf{H}_{s}\right)=\left(2 M_{R}+1\right) \log (\pi e)+\log |\boldsymbol{\Gamma}|,
$$

where $h(\cdot)$ denotes the entropy function and the logarithms are all in base 2. Since $\mathbf{H}_{s}$ is complex, $\log (\pi e)$ is used in (13) instead of the usual $\log (2 \pi e)$. It is instructive to examine the limiting behavior of the entropy $h\left(\mathbf{H}_{s}\right)$ defined as

$$
h_{\infty}\left(\mathbf{H}_{s}\right) \triangleq \lim _{M_{R} \rightarrow \infty} \frac{h\left(\mathbf{H}_{s}\right)}{\left(2 M_{R}+1\right)} .
$$

It is noted that according to (9), the size of vector $\mathbf{H}_{s}$ is directly related to the radius of the receiver region $r_{R}$.
Therefore, $M_{R} \rightarrow \infty$ is equivalent to choosing an infinitely large radius to place the receiver antennas. Using (11)-(13) and the known results on the entropy rate of Gaussian processes [12, pp. 273-274], we obtain the following theorem.

Theorem 1: (Random Scattering Entropy Rate) The entropy rate of a random scattering process around an infinitely large receiver radius $r_{R}$ is given as

$$
h_{\infty}\left(\mathbf{H}_{s}\right)=\log (\pi e)+\frac{1}{2 \pi} \int_{0}^{2 \pi} \log P(\phi) d \phi
$$

where $P(\phi)$ is the angular power spectrum incident on the receiver region, which was defined in (10).

Two important corollaries follow from the above theorem. First, suppose that $P\left(\phi^{\prime}\right)=0$ for some $\phi^{\prime} \in[0,2 \pi)$. This results in $\log P(\phi) \rightarrow-\infty$ and causes the entropy rate $h_{\infty}\left(\mathbf{H}_{s}\right) \rightarrow-\infty$. This analysis assumes that $\int \log P(\phi) d \phi$ is finite for $\phi \neq \phi^{\prime}$ and also excludes special cases, where $\log (\cdot)$ is integrable from 0 to $2 \pi$ (an example is $\int_{0}^{2 \pi} \log (x) d x$ ). It should be emphasized that $h\left(\mathbf{H}_{s}\right)$ and $h_{\infty}\left(\mathbf{H}_{s}\right)$ are differential entropies and both positive and negative numbers are allowed [12, Ch. 9]. $h_{\infty}\left(\mathbf{H}_{s}\right) \rightarrow-\infty$ would mean that $\mathbf{H}_{s}$ is no longer random and may be considered as deterministic. This discussion is summarized in the following corollary.

Corollary 1.1: (Entropy Rate of Directional Angular Power Spectrum) If the angular power spectrum $P(\phi)$ is bandlimited or directional, i.e. if $P(\phi)$ contains a null in the region $[0,2 \pi)$, then the observed scattering at a sufficiently large receiver region is no longer random.

Similarly, the following corollary states the necessary condition for the scattering process to retain its randomness, even for large receiver radii.

Corollary 1.2: (Purely Random Scattering) In order to have random scattering observed at sufficiently large receiver region, the angular power spectrum $P(\phi)$ should not contain any nulls in the whole region $[0,2 \pi)$.

Before, we present the numerical analysis, it is instructive to consider two special cases of 1) uniform isotropic scattering and 2) a unidirectional power spectrum.

Special Case 1: (Uniform Isotropic Scattering) In this case, $P(\phi)=1 / 2 \pi$ everywhere and its Fourier series coefficients are $\gamma_{m}=0$ for $m \neq 0$ and $\gamma_{0}=1$. Therefore, the Gaussian r.v's in $\mathbf{H}_{s}$ are independent regardless of its size $\left(2 M_{R}+1\right)$ and a purely random scattering is observed.

Special Case 2: (Unidirectional Power Spectrum) In this case, $P(\phi)=\delta\left(\phi-\phi^{\prime}\right)$ for some $\phi^{\prime} \in[0,2 \pi)$, where $\phi^{\prime}$ is direction of the single plane-wave and $\delta(\cdot)$ is the Dirac's delta function. The Fourier series coefficients are given as $\gamma_{m}=e^{j \phi^{\prime}}, \forall m$. Therefore, the Gaussian r.v's in $\mathbf{H}_{s}$ are purely correlated and their covariance matrix $\Gamma$ becomes singular for $M_{R}>1$. As a result, the scatterer is observed as deterministic $\left(h\left(\mathbf{H}_{s}\right) \rightarrow-\infty\right)$ for any receiver radius $r_{R}>0$. 


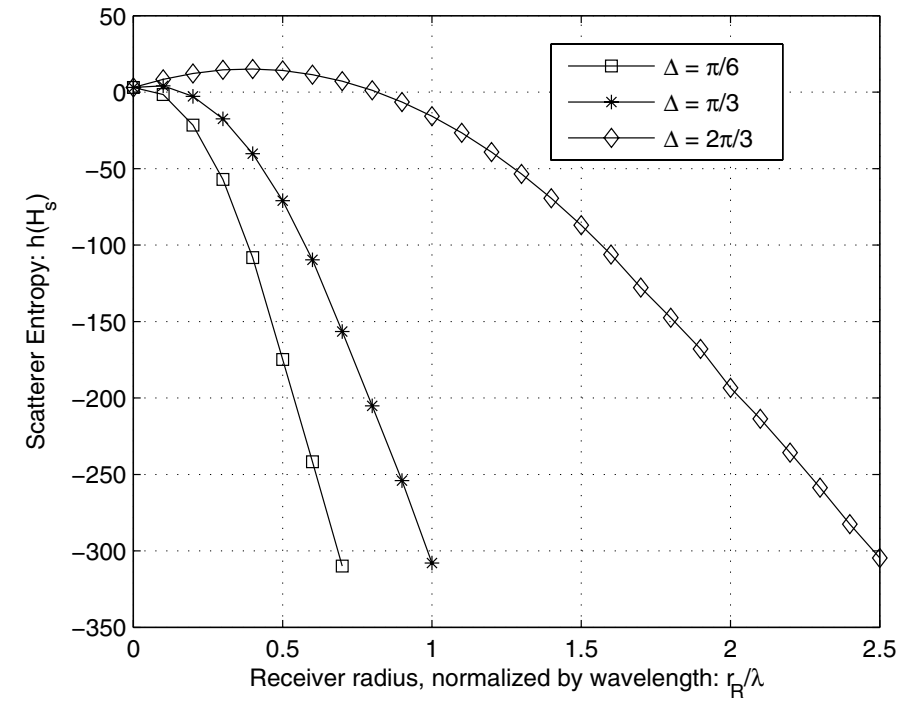

Fig. 1. Random scatterer entropy $h\left(\mathbf{H}_{s}\right)$ as a function of normalized receiver region radius $r_{R}$ for various uniformly directional scatterers. A more directional scatterer field (smaller $\Delta$ ) is less random than a less directional scatterer field (larger $\Delta$ ). The scattering randomness rapidly decreases with increasing the receiver radius. For each scatterer, there is a receiver radius with the maximum observed scattering randomness.

\section{Numerical Analysis}

Here, we analyze the entropy of uniformly directional random scatterers as a function of receiver region radius. More specifically, we assume that the energy is arriving at the receiver scatterer-free region in a restricted range: $P(\phi)=$ $1 / 2 \Delta, \phi \in[-\Delta, \Delta]$. The elements of the covariance matrix $\Gamma$ for $\mathbf{H}_{s}$ in (11) and (12) are given as [14]

$$
\gamma_{m}=\operatorname{sinc}(m \Delta)
$$

where $\operatorname{sinc}(x) \triangleq \sin (x) / x$ for $x \neq 0$ and $\operatorname{sinc}(0) \triangleq 1$. Fig. 1 shows the random scatterer entropy $h\left(\mathbf{H}_{s}\right)$ in (13) as a function of receiver radius $r_{R}$ and $\Delta$. Note that the random scatterer size $\left(2 M_{R}+1\right)$ is related to $r_{R}$ through (9). It is observed from this figure that random scattering entropy rapidly decreases as the observer (receiver) radius increases. As expected, a more directional scatterer field (smaller $\Delta$ ) is less random than a less directional scatterer field (larger $\Delta$ ). Moreover, for each angular spread, there is a receiver radius with the maximum scattering randomness.

Evaluating random scatterer entropy using (12) and (13) is generally applicable for any desired angular power spectrum (other than uniform), such as those provided in [14].

\section{MIMO RECEIVER ANTENNA CONFIGURATION}

\section{A. Predictive Analysis}

In this section, we are interested to find the effect of MIMO receiver antenna configuration (such as receiver region radius $r_{R}$, number of receiver antenna elements $N_{R}$, and antenna arrangement) on the MIMO channel covariance matrix. In Section III, we analyzed the random behavior of the scattering vector $\mathbf{H}_{s}$. In view of (6), the channel matrix $\mathbf{H}=\mathbf{J}_{R} \mathbf{H}_{s}$ is a linear transformation of scattering r.v's in $\mathbf{H}_{s}$.
We examine the receiver antenna configuration matrix $\mathbf{J}_{R}$ in more detail. Assume that the $n^{\text {th }}$ receiver antenna element is positioned at a distance $z_{n} \leqslant r_{R}$ from the origin of the receiver scatterer-free region and its angle from the azimuth reference is $\psi_{n}$. Then, $\mathbf{J}_{R}$ is an $N_{R} \times\left(2 M_{R}+1\right)$ matrix, which is written as [11]

$\mathbf{J}_{R}=\left[\begin{array}{ccc}a_{-M_{R}}\left(z_{1}\right) e^{i M_{R} \psi_{1}} & \cdots & a_{M_{R}}\left(z_{1}\right) e^{-i M_{R} \psi_{1}} \\ \vdots & \ddots & \vdots \\ a_{-M_{R}}\left(z_{N_{R}}\right) e^{i M_{R} \psi_{N_{R}}} & \cdots & a_{M_{R}}\left(z_{N_{R}}\right) e^{-i M_{R} \psi_{N_{R}}}\end{array}\right]$

where $a_{m}\left(z_{n}\right) \triangleq J_{m}\left(k z_{n}\right) e^{i m \pi / 2}$ and $J_{m}(\cdot)$ is the $m^{t h}$-order Bessel function of the first kind. Also, $M_{R}$ was related to the receiver radius $r_{R}$ through (9).

According to Proposition 1 in Section II, we are interested in analyzing $|\mathbf{W}|$, where $\mathbf{W} \triangleq \frac{\eta}{N_{T}} E_{\mathbf{H}}\left\{\mathbf{H} \mathbf{H}^{H}\right\}$. Using (6), $\mathbf{W}$ is written as

$$
\mathbf{W}=\frac{\eta}{N_{T}} \mathbf{J}_{R} E\left[\mathbf{H}_{s} \mathbf{H}_{s}^{H}\right] \mathbf{J}_{R}^{H}=\frac{\eta}{N_{T}} \mathbf{J}_{R} \boldsymbol{\Gamma} \mathbf{J}_{R}^{H},
$$

where $\Gamma$ is the scattering covariance matrix, with elements defined in (12). Furthermore,

$$
|\mathbf{W}|=\left(\frac{\eta}{N_{T}}\right)^{N_{R}}\left|\mathbf{J}_{R} \boldsymbol{\Gamma} \mathbf{J}_{R}^{H}\right| .
$$

From (19), the effect of receiver antenna configuration matrix $\mathbf{J}_{R}$ on $|\mathbf{W}|$ becomes clear. In [11], the effects of receiver radius $r_{R}$ and the number of receiver antenna elements $N_{R}$ on $|\mathbf{W}|$ were discussed. Here, we review these results and provide a second proposition on the optimum receiver antenna arrangement, which is the contributions of this paper.

1) Increasing the number of receiver antennas for a fixed receiver radius: For a fixed receiver radius $r_{R}$, there are $\left(2 M_{R}+1\right)$ r.v's in the scattering vector $\mathbf{H}_{s}$. Increasing the number of receiver antennas beyond $N_{R}>\left(2 M_{R}+1\right)$ in $\mathbf{J}_{R}$ results in linearly dependent elements in $\mathbf{H}$. Hence, the covariance matrix $\mathbf{W}$ becomes singular. So, the rank of $\mathbf{W}$ is limited by $\left(2 M_{R}+1\right)$.

Remark: (Directional Scattering) We observed in Section III that the entropy of directional scattering approaches $-\infty$ for large receiver radii. This means that the scattering vector $\mathbf{H}_{s}$ will no longer be full rank. Therefore, for large receiver radii, we expect a singular $\mathbf{W}$ even for $N_{R}<\left(2 M_{R}+1\right)$. This, in turn, will limit the expected linear increase of MIMO capacity with increasing the number of antennas.

2) Optimum receiver antenna arrangement: A relevant question that arises is that for a given random scattering environment, which is characterized by its angular power spectrum $P(\phi)$, how the receiver antenna elements should be arranged to maximize MIMO capacity. Based on Proposition 1 in Section II, maximizing $|\mathbf{W}|$ is an accurate approach for $N_{T}>N_{R}$ and uncorrelated transmitted branches. According to the Hadamard's inequality in (4), making the MIMO channel covariance matrix $\mathbf{W}$ diagonal will maximize $|\mathbf{W}|$. With no constraint imposed on the receiver configuration matrix $\mathbf{J}_{R}$ and referring to (18), $\mathbf{J}_{R}$ should be chosen as the eigenvectors (or singular value decomposition matrices) of the 


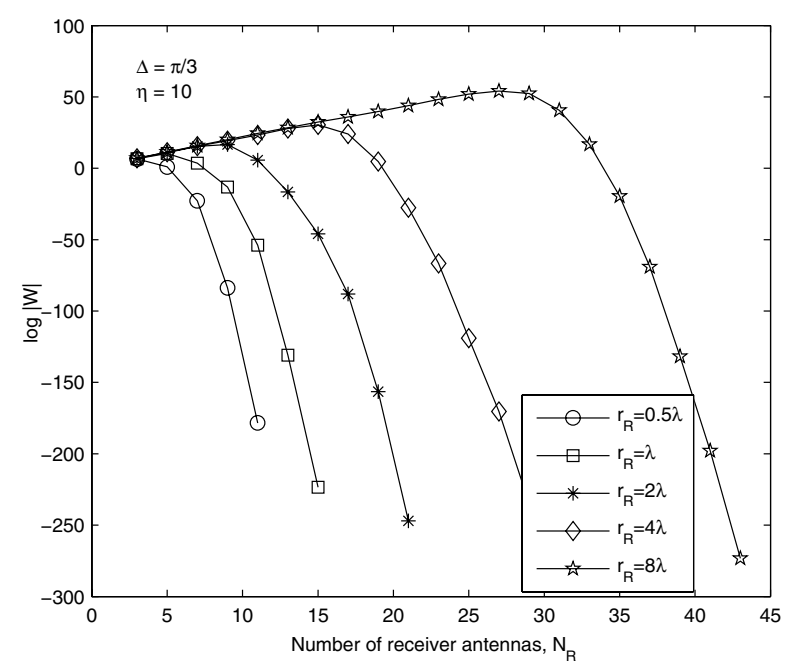

Fig. 2. $|\mathbf{W}|$ given in (19) in logarithmic scale as a function of number of receiver antenna elements $N_{R}$ and receiver radii $r_{R}$. For a fixed receiver radius, increasing the number of receiver antennas first results in an increase in $|\mathbf{W}|$. Then, $|\mathbf{W}|$ decreases due to the linear dependence introduced by the receiver configuration matrix $\mathbf{J}_{R}$.

random scatterer covariance matrix $\boldsymbol{\Gamma}$. However, the receiver configuration matrix $\mathbf{J}_{R}$ in (17) has a constrained structure. Therefore, maximizing MIMO capacity by means of proper receiver antenna arrangement is equivalent to the constrained convex optimization of $|\mathbf{W}|$ subject to the structure of $\mathbf{J}_{R}$.

\section{B. Numerical Analysis}

We first study the effect of increasing the number of receiver antenna elements $N_{R}$ on $|\mathbf{W}|$ defined in (19). Fig. 2 shows $|\mathbf{W}|$ in logarithmic scale as a function of number of receiver antenna elements $N_{R}$ for various receiver radii $r_{R}$. The angular power spectrum $P(\phi)$ is uniformly non-zero in the range $\phi \in[-\Delta, \Delta]$ and $\Delta=\pi / 3$ is chosen. SNR is fixed to $\eta=10$. Receiver antennas are circularly located at $z_{R}=r_{R}$ and are also uniformly arranged in the interval $\phi \in[-\Delta, \Delta]$, where the angular power spectrum is non-zero. There are a number of points that can be concluded from Fig. 2 as follows.

- Increasing the number of receiver antennas first results in an increase in $|\mathbf{W}|$. However, as predicted above, due to the linear dependence introduced by $\mathbf{J}_{R}, \mathbf{W}$ becomes singular by further increase in the number of receiver antenna elements and hence, $|\mathbf{W}|$ decreases.

- It is observed that for each receiver radius, there is an optimum number of receiver antenna elements $N_{R}^{*}$ that maximizes $|\mathbf{W}|$. The ratio $N_{R}^{*} /\left(2 M_{R}+1\right)$ is around 0.19 to 0.27 and decreases with increasing the receiver radius.

- Table I shows the optimum normalized receiver antenna separation for $|\mathbf{W}|_{\max }$. From this table it is concluded that the optimum antenna separation is almost insensitive to the receiver radius and is very close to the well-known $\lambda / 2$ separation rule of thumb.

Next, we study the effect of receiver antenna arrangement on $|\mathbf{W}|$. Fig. 3 shows $|\mathbf{W}|$ in logarithmic scale for various receiver antenna arrangements. The angular power spectrum is

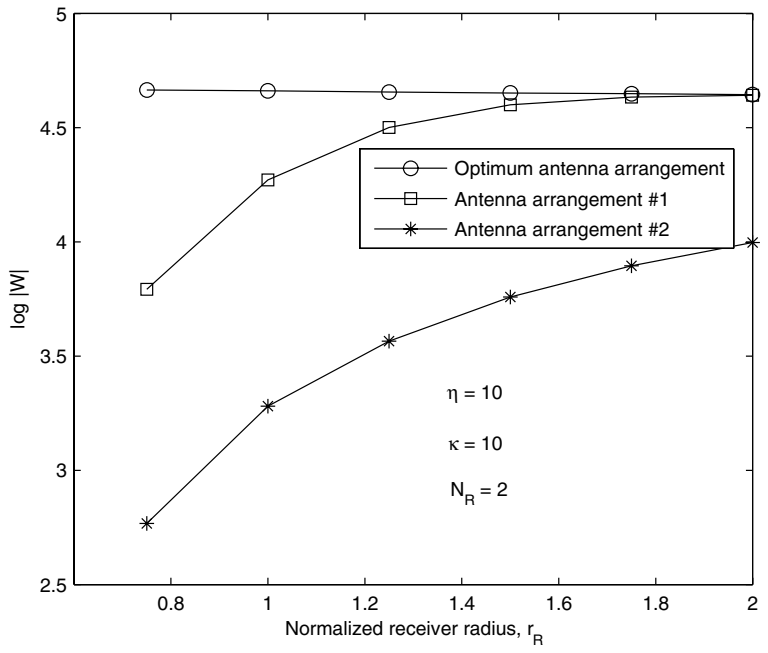

Fig. 3. $|\mathbf{W}|$ given in (19) in logarithmic scale as a function of receiver radius $r_{R}$ and antenna arrangement. The angular power spectrum is non-uniform and highly directional and follows a von-Mises spectrum [14] with $\kappa=10$. It is possible to maximize $|\mathbf{W}|$ by proper antenna arrangement.

non-uniform and highly directional and follows a von-Mises distribution [14] with $\kappa=10$. The number of receiver antennas is $N_{R}=2$. Two fixed receiver antenna arrangements are shown (numbered as 1 and 2 in the figure). In the first setting, receiver antennas are circularly located at $z_{R}=r_{R}$ and are positioned at $\psi_{1}=0.1 \pi$ and $\psi_{2}=-0.1 \pi$. In the second setting, antennas are positioned at the maximum antipodal distance from each other $\left(\psi_{1}=0\right.$ and $\left.\psi_{2}=\pi\right)$. However, this setting ignores the directional nature of scatterer. The upper graph corresponds to the receiver antenna positions that maximize $|\mathbf{W}|$ (subject to the constraint in $\mathbf{J}_{R}$ and also $z_{1}=z_{2}=r_{R}$ ). The optimum antenna position is found to be $\psi_{1}=0.3 \pi$ and $\psi_{2}=-0.3 \pi$.

\section{MIMO CAPACITY ANALYSIS}

In previous sections, we analyzed $|\mathbf{W}|$ or its components, without direct analysis of MIMO channel capacity in (2) or (3). In this section, we study the effect of directional scattering, which is represented in the spatial decomposition of the channel matrix in (6), on MIMO channel capacity.

First, we study the effect of increasing the number of receiver antenna elements $N_{R}$ on the MIMO capacity upper bound in (3). We assume that $N_{T}>>N_{R}$ and the transmitted branches are uncorrelated, so that the upper bound (3) is an accurate estimate of channel capacity. The parameters for capacity analysis are chosen to be identical to those in Fig. 2 . Receiver antennas are circularly located at $z_{R}=r_{R}$ and are also uniformly arranged in the interval $\phi \in[-\Delta, \Delta]$, where the angular power spectrum is non-zero. Fig. 4 shows the MIMO capacity upper bound. By comparing Fig. 2 and Fig. 4 it is concluded that the knee points of MIMO capacity in Fig. 4 coincide with the points where $|\mathbf{W}|$ is maximized in Fig. 2.

Next, we find the optimum antenna arrangement by direct evaluation of MIMO channel capacity in (2) for $N_{T}=1$ and $N_{R}=2$. Fig. 5 shows the Monte-Carlo estimates of 


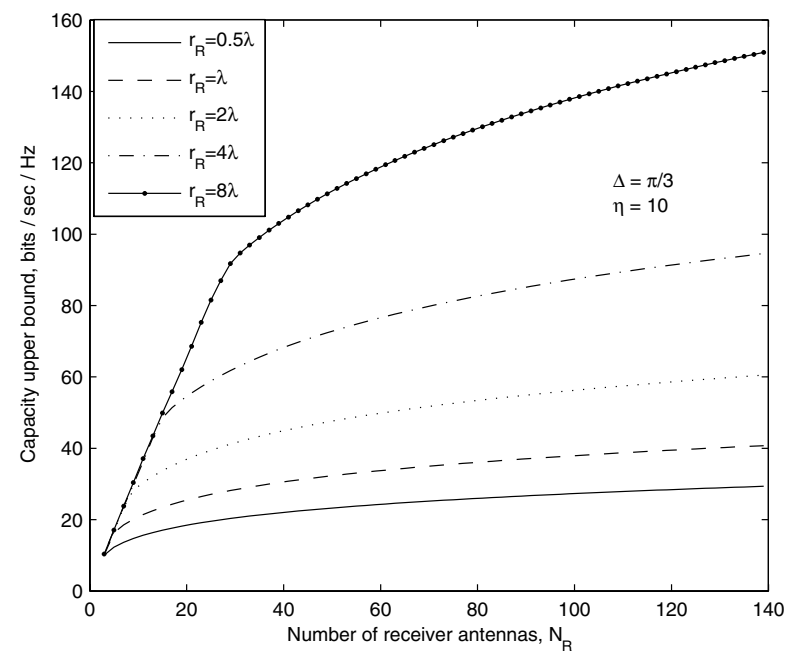

Fig. 4. MIMO capacity upper bound in (3) as a function of the number of receiver antenna elements $N_{R}\left(N_{T}>>N_{R}\right)$. The uniformly directional scatterer and antenna arrangements are identical to Fig. 2. The knee points in the upper bound coincide with the points where $|\mathbf{W}|$ in Fig. 2 is maximized.

TABLE I

THE OPTIMUM SEPARATION OF RECEIVER ANTENNA ELEMENTS THAT MAXIMIZES $|\mathbf{W}|$ IN FIG. 2.

\begin{tabular}{|c|c|c|c|c|c|}
\hline Receiver radius, $r_{R}$ & $0.5 \lambda$ & $\lambda$ & $2 \lambda$ & $4 \lambda$ & $8 \lambda$ \\
\hline Antenna distance & $0.50 \lambda$ & $0.52 \lambda$ & $0.52 \lambda$ & $0.60 \lambda$ & $0.64 \lambda$ \\
\hline
\end{tabular}

the MIMO capacity in (2) for various antenna arrangements. Other simulation settings are identical to Fig. 3. It is verified from this figure that the optimum antenna arrangement that maximizes $|\mathbf{W}|$ in Fig. 3 also results in the highest MIMO capacity, even for the case $N_{T}=1$. In particular, the capacity enhancement using the optimum antenna arrangement is noticeable compared to the antenna setting \#2, where antenna elements are antipodal $\left(\psi_{1}=0\right.$ and $\left.\psi_{2}=\pi\right)$ and ignore the directional nature of the scattering environment.

\section{CONCLUSIONS}

Directional or bandlimited random scattering poses a fundamental limit on the capacity of MIMO systems. In this paper, we studied this limit by analyzing the inherent randomness (entropy) of directional scatterers using the spatial decomposition of the MIMO channel matrix. We showed that directional scatterers (with at least a null in the angular power spectrum) will no longer be random when the receiver observation radius is sufficiently large. We also studied the effect of receiver antenna arrangement on MIMO capacity. We showed that for any random scattering with a given angular power spectrum, it is possible to find the optimum receiver antenna arrangement that maximizes MIMO channel capacity. The optimum receiver antenna arrangement transforms the random scatterers to minimize the correlation in the MIMO channel matrix, subject to the electromagnetic constraints on the receiver antenna configuration matrix.

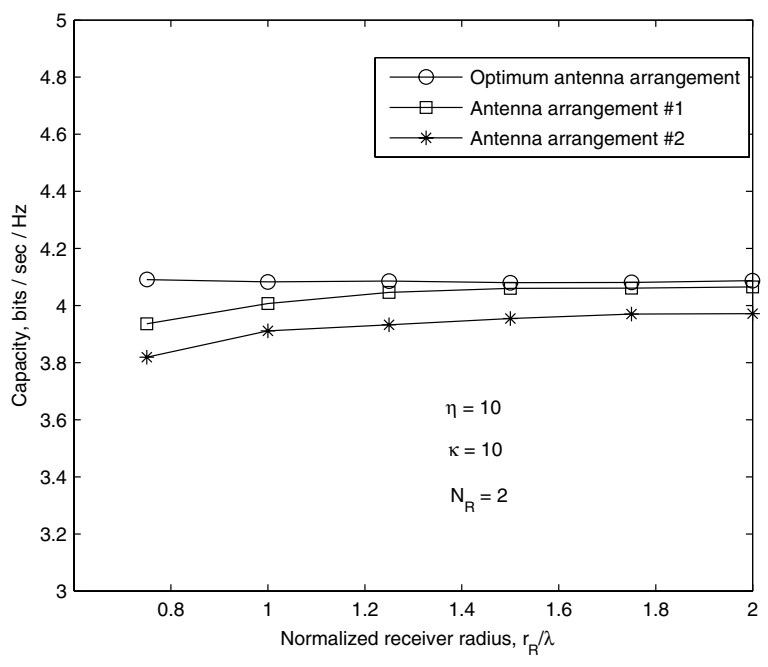

Fig. 5. MIMO capacity in (2) as a function of receiver radius $r_{R}$ for three receiver antenna arrangements. The directional scatterer and receiver antenna arrangements are identical to Fig. 3. It is observed that the optimum antenna arrangement that maximizes $|\mathbf{W}|$ in Fig. 3 also results in the highest MIMO capacity.

\section{REFERENCES}

[1] I. E. Telatar, "Capacity of multiple-antenna Gaussian channels," Eur. Trans. Telecom., vol. 10, pp. 585-595, Nov. 1999.

[2] G. J. Foschini and M. J. Gans, "On the limits of wireless communications in a fading environment when using multiple antennas," Wireless Personal Communications, vol. 6, no. 2, pp. 311-355, 1998.

[3] X. Mestre, J. R. Fonollosa, and A. Pagès-Zamora, "Capacity of MIMO channels: asymptotic evaluation under correlated fading," IEEE J. Select. Areas Commun., vol. 21, no. 5, pp. 829-838, June 2003.

[4] C. N. Chuah, D. Tse, J. M. Kahn, and R. A. Valenzuela, "Capacity scaling in MIMO wireless systems under correlated fading," IEEE Trans. Inform. Theory, vol. 48, no. 3, pp. 637-650, Mar. 2002.

[5] C. Martin and B. Ottersen, "Asymptotic eigenvalue distributions and capacity for MIMO channels under correlated fading," IEEE Trans. Wireless Commun., vol. 3, no. 4, pp. 1350-1359, July 2004.

[6] H. Bölcskei, M. Borgmann, and A. J. Paulraj, "Impact of the propagation environment on the performance of spaced-frequency coded MIMOOFDM," IEEE J. Select. Areas Commun., vol. 21, no. 3, pp. 427-439, Apr. 2003.

[7] H. Bölcskei and A. J. Paulraj, "Performance of space-time codes in the presence of fading correlation," in Proc. Asilomar Conf. Signals Systems and Computers, Oct. 2000, pp. 687-693.

[8] T. S. Pollock, T. D. Abahayapala, and R. A. Kennedy, "Introducing space into MIMO capacity calculations," Journal on Telecom. Systems, vol. 24, no. 2, pp. 415-436, 2003.

[9] R. R. Muller, "A random matrix model of communication via antenna arrays," IEEE Trans. Inform. Theory, vol. 48, no. 9, pp. 2495-2506, Sept. 2002.

[10] M. Debbah and R. R. Muller, "MIMO channel modeling and the principle of maximum entropy," IEEE Trans. Inform. Theory, vol. 51, no. 5, pp. 1667-1690, May 2005.

[11] T. D. Abahayapala, T. S. Pollock, and R. A. Kennedy, "Spatial decomposition of MIMO wireless channels," in Proc. Int. Symp. Signal Processing and Its Applications, July 2003, pp. 309-312.

[12] T. M. Cover and J. A. Thomas, Elements of Information Theory, 1st ed. New York: Wiley, 1991.

[13] C. Oestges, B. Clreckx, D. Vanhoenacker-Janvier, and A. J. Paulraj, "Impact of fading correlations on MIMO communication systems in geometry-based statistical channel models," IEEE Trans. Wireless Commun., vol. 4, no. 3, pp. 1112-1120, May 2005.

[14] P. S. Teal, T. D. Abahayapala, and R. A. Kennedy, "Spatial correlation for general distribution of scatteres," IEEE Signal Processing Lett., vol. 9, no. 10, pp. 305-308, Oct. 2002. 